\title{
DESIGN OF A VIRTUAL COMMUNITY INFRASTRUCTURE FOR ELDERLY CARE
}

\author{
L. M. Camarinha-Matos ${ }^{l}$ and H. Afsarmanesh ${ }^{2}$ \\ 'New University of Lisbon, PORTUGAL \\ Quinta da Torre, 2829 Monte Caparica, Portugal, cam@uninova.pt \\ ${ }^{2}$ University of Amsterdam, THE NETHERLANDS \\ Kruislaan 403, 1098 SJ Amsterdam, The Netherlands, hamideh@wins.uva.nl
}

The virtual organizations paradigm combined with adequate tools to support the cooperation of social care assistants, health care professionals, elderly people, and their relatives, constituting a "virtual community", represent a promising approach to reduce the overwhelming care provision costs and to increase their quality. An architecture for a flexible platform based on adaptive mobile agents and information federation mechanisms is proposed and preliminary feasibility experiments are described.

\section{INTRODUCTION}

The concept of virtual organization (VO) initially more focused on the interenterprise cooperation, is now spreading to other less traditional domains such as the elderly care sector. An integrated elderly care system comprises a number of organizations such as social security institutions, care centers / day centers, health care institutions, etc. and involves the cooperation of a number of different human actors e.g. social care assistants, health care professionals, the elderly people and their relatives. If supported by computer networks and adequate supporting tools such systems may evolve towards operating as a long-term virtual organization and the various involved actors become part of a virtual community (VC).

Finding more effective ways of providing care to a growing number of elderly became a major challenge in Europe. In fact during the last three decades, the number of people aged from 60 years or more has risen about $50 \%$ [8]. Today, more than $25 \%$ of Europe's population is aged 60 years or more and this trend will continue. Within 20 years approximately a third of Europe's population will be over 60 years old, a total in excess of 100 million citizens. The percentage of people over the age of 80 is growing even more rapidly: an increase of $300 \%$ has been forecasted for the period of 1960 to 2020 . Moreover, the ratio between the working and elderly population is dramatically declining. In 1995 there were almost seven adults of working age per one elderly person (aged 65 or more), by 2025 the ratio is expected to decrease to close to four. Similarly in the USA, it is expected that in 2020 about 1 in 6 Americans will be elderly. These trends present a real challenge for the social policies in the years ahead and underline the importance of developing new and more cost-effective ways of and facilities providing care and support to the elderly.

Furthermore, as a group in society, the elderly face the real threat of being left out and excluded from the benefits of further European integration in general, and

\footnotetext{
The original version of this chapter was revised: The copyright line was incorrect. This has been corrected. The Erratum to this chapter is available at DOI: 10.1007/978-0-387-35585-6_68
} 
indeed the internal market in particular. New approaches and ideas are required, and steps need to be taken to provide them with the means to exercise their freedom of choice and independence.

Traditional approach to care provision has either resorted to support from the relatives, or from the elderly care centres. However, these two solutions have become increasingly inappropriate for the following reasons:

(i) Shifting the burden of responsibility onto relatives is increasingly impractical, given the fact that more and more family members have to work to secure steady incomes.

(ii) Provision of enough care centers is costly and invariably necessitates the relocation of the elderly people, often beyond their home communities.

(iii) Many elderly people preserve enough robustness to be in their homes, a situation that is often preferable to them, and as such better for their welfare.

Various research projects have been trying to develop technological solutions to increase the care services and reduce their costs [8]. For instance, various experiments have installed "social alarm" systems, namely in the case of people living in remote places. Such systems comprise a portable alarm trigger and an alarm telephone that dials a social alarm control center when there is any emergency situation [10]. More recent works are focused on mobile social alarm systems and monitoring systems based on a diversity of sensors and other devices. Advances in computer networks and ubiquitous computing suggest the opportunity for more advanced care approaches including comprehensive status monitoring, other forms of assistance such as reminders, but also the creation of the opportunity for the elderly becoming involved in a community, i.e. reducing their feeling of loneliness.

In this context, the recently launched IST TeleCARE project [9] aims at design and development of a configurable framework focused on virtual communities for elderly support. The proposed solution has to be seen as complementary to other initiatives for the integration of elderly in the society and to reduce their isolation. The TeleCARE consortium involves research organizations, technology developers, socio-economic experts, and elderly support organizations from Portugal, Spain, UK, and the Netherlands.

\section{AN EXAMPLE SCENARIO}

The objective of TeleCARE is to leverage the potential of information society technologies, in particular by resorting to the virtual organizations and multi-agent systems paradigms, to improve the quality of life and care, for elderly people and their families. Important requirements for this sector, in opinion of TeleCARE end users, include the following aspects:

- Guarantee of a high degree of security and privacy for users, and the welfare considerations for the elderly.

- Improvement of the costs, quality, and accessibility of the care services.

- The quality of life of the elderly users must be ensured by adequate support not only for health care, but also for their involvement in leisure/social activities.

- The privacy, ethical and socio-organizational issues must be considered when developing any such technical solutions. 
In TeleCARE, an open infrastructure supporting the cooperation of various cooperative entities is planned (see example in Fig. 1). Different classes of software agents are designed to perform a large number of activities. Such activities include: (1) local and remote supervision tasks: such as sensorial data collection, health conditions monitoring, and alarm notification; (2) intelligent assistance: such as agenda assistance, and leisure activities assistance; and (3) integration/federation and provision of necessary data from distributed sources in the network: such as collection and preparation of health related data for elderly.

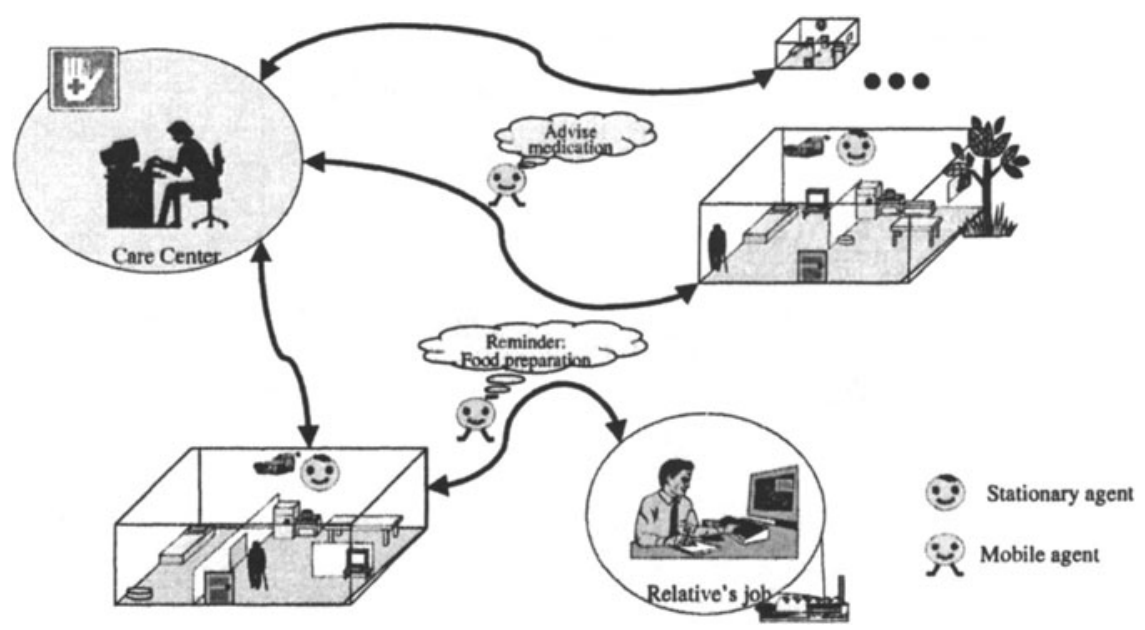

Figure 1 - An example scenario for the TeleCARE infrastructure

In order to better understand the scope of TeleCARE, let us consider a typical situation:

"John Smith is 70 years old and he suffers from diabetes and locomotion problems. He is the father of Mary and he is affiliated with the ALFA-team Care Center."

Various episodes of the daily life of different actors in this situation are shown in Fig.2. Some important facets can be derived from this example:

- The elderly, probably by the initiative of a relative, subscribes to the services of the ALFA-team network of care organizations. The joining process will specify the duties and rights of all parties.

- Monitoring sensors will be installed at the elderly's home; and home appliances will be "intelligent" and connected to a computer network.

- Intelligent software agents (stationary and mobile) are used to perform a variety of missions (services) e.g. status monitoring, reminding actions, assistance missions, integrated data collection, etc.

- Software agents sent from one node (e.g. care center) to others (e.g. different homes) must adapt themselves to the local resources and environment that they find at each node.

In addition to these basic characteristics, an advanced care system should offer the elderly the opportunity to:

-Feel connected to the rest of the world, and feel not left alone; 
-Get involved in community activities and leisure activities, with other elderly;

-Be recognized for his/her contribution;

-Participate in events (even without moving from home), or even telework;

-Be able to leave home (visit friends or go for a walk), and whenever needed receive some medical support;

-Be reminded of important issues.

The envisaged scenario requires a generic open (horizontal) infrastructure supporting the formation of a virtual organization / virtual community and its basic interaction mechanisms. On top of this horizontal infrastructure an extensive number of specialized (vertical) services could be developed.

\begin{tabular}{|c|c|}
\hline प. & $\begin{array}{l}\text { In the morning (wake up) } \\
\text { - There is an assistant agent who reminds } \\
\text { Mr. Smith about the daily medication, and } \\
\text { other possible actions: e.g., put the sensor } \\
\text { wristband; use the Spiro meter to check his } \\
\text { vital signs, etc. } \\
\text { - Monitor agent sends detailed health-data } \\
\text { of Mr. Smith to the ALFA-team Care Center } \\
\text { and a summary to his daughter Mary. }\end{array}$ \\
\hline $\begin{array}{l}\text { At noon (lunch time) } \\
\text { - An agent controls the microwave to warm } \\
\text { up the lunch (that was left there in the } \\
\text { morning by Mary), or } \\
\text { - An agent that carries a plan, or instructions } \\
\text { to prepare Mr. Smith's lunch, arrives at the } \\
\text { elderly's home. } \\
\text { - Also, in an ideal situation, an agent checks } \\
\text { the content of the fridge, and other places, } \\
\text { like pantry, and sends this information to the } \\
\text { Care Center and to Mary, or use this } \\
\text { information to prepare the lunch by itself. }\end{array}$ & (I) \\
\hline 맘ㅁㄹ & $\begin{array}{l}\text { When there is an emergency } \\
\text { - A sensor agent (in the wristband) informs } \\
\text { a supervisor agent about an anomaly, and } \\
\text { sends an alarm to both the ALFA-team Care } \\
\text { Center and to Mary. } \\
\text { - Another agent tries to verify the alarm, } \\
\text { activating other agents (e.g., camera agent) } \\
\text { and determines the seriousness of the } \\
\text { situation (issue reconfirmation information). } \\
\text { - In case of a serious situation, the } \\
\text { supervisor agent sends an alarm and } \\
\text { data/information of the vital signals of Mr. } \\
\text { Smith directly to the ALFA-team Health } \\
\text { Center. }\end{array}$ \\
\hline
\end{tabular}

Figure 2 - Episodes of an example scenario 
Besides the elderly, a large number of actors can be part of a virtual elderly care community, including: 1) Care providers - social care assistants, doctors, and other health care professionals, as well as the officers from the fire department or police stations; 2) Relatives of the elderly - either living with the elderly, but being at work, in a vehicle, etc., or living in another house; and 3) Other elderly - wishing to get involved in joint (leisure) activities.

\section{ARCHITECTURE DESIGN PRINCIPLES}

Elderly care is a new area for the application of the VO/VC concepts, and a domain that can be characterized by:

- a market that is still incipient and mostly targeted by SMEs, and

- a wide variety of opportunities and promises suggested by the new technologies (e.g. the house of the future, with wireless computerized appliances) that still need to be tested, namely in terms of their performance and acceptance by the various actors.

Considering these characteristics, it is important to design a generic, open, and configurable horizontal infrastructure, that is both flexible enough to adapt to the new market developments, and on top of which new vertical services can progressively be created. It is clear that a large level of heterogeneity is likely to be found in each home - since different people have different need and possess different hardware infrastructures, and a wide range of diversity and autonomy among other nodes within the virtual elderly care community. Therefore, it is important that the virtual care community (VCC) infrastructure for TeleCARE be layered, separating the general / common functionalities from the specific aspects of each site.

Fig. 3 shows a first proposal for the architecture of a platform that will be installed in each node of the care organization.

The fundamental elements of the basic platform include:

- Safe communications infrastructure that provides safe communications; in critical cases where communications reliability is mandatory redundant channels, in addition to Internet, may be supported.

- Interfaces to sensors / devices, monitoring devices and other hardware (home appliances, environment controllers, etc.). These interfaces represent the bridge to any "intelligent home" or "local domotic network" hiding aspects such as low-level protocols, wire-based or wireless communications, etc.

- Core Multi-agent platform, that is the main component of the basic platform. It supports the creation, launching, reception (authentication and rights verification), and execution of stationary and mobile agents as well as their interactions. As intelligent agents are envisaged, an inference engine (e.g. JESS or Prolog interpreter) is included. A neutral registry (catalog) of all available services / resources is a key element to support a flexible infrastructure. Main modules in this layer:

a. Basic multi-agent platform (e.g. JADE or AGLETS).

b. Inference engine (e.g. JESS or Prolog interpreter). 
c. Inter-platform mobility - Extension to the basic MAS platform to support generalized mobility of agents.

d.Inter-agent communication - Extension to the basic MAS platform to support communication between / coordination of agents independently of their local.

e. Agent factory - module that supports the creation / specification and launching of new agents. It can be based on a catalog of agent types (agent skeletons).

f. Platform manager - To configure and specify the operating conditions of the platform in each site, recover from errors, monitor operation, etc.

g. Local federated information manager, to support the necessary local information management while preserving the information privacy and careful control of access rights to local data for external users. This modules, installed in each site, is the local component of the distributed / federated agile information management system.

h. Agent reception and registration - To accept incoming mobile agents and grant them with the authorized permissions (generation of local passport).

i. Resource manager - To manage the catalogue of resources including the specification (and access proxies) of all services available at the current site.

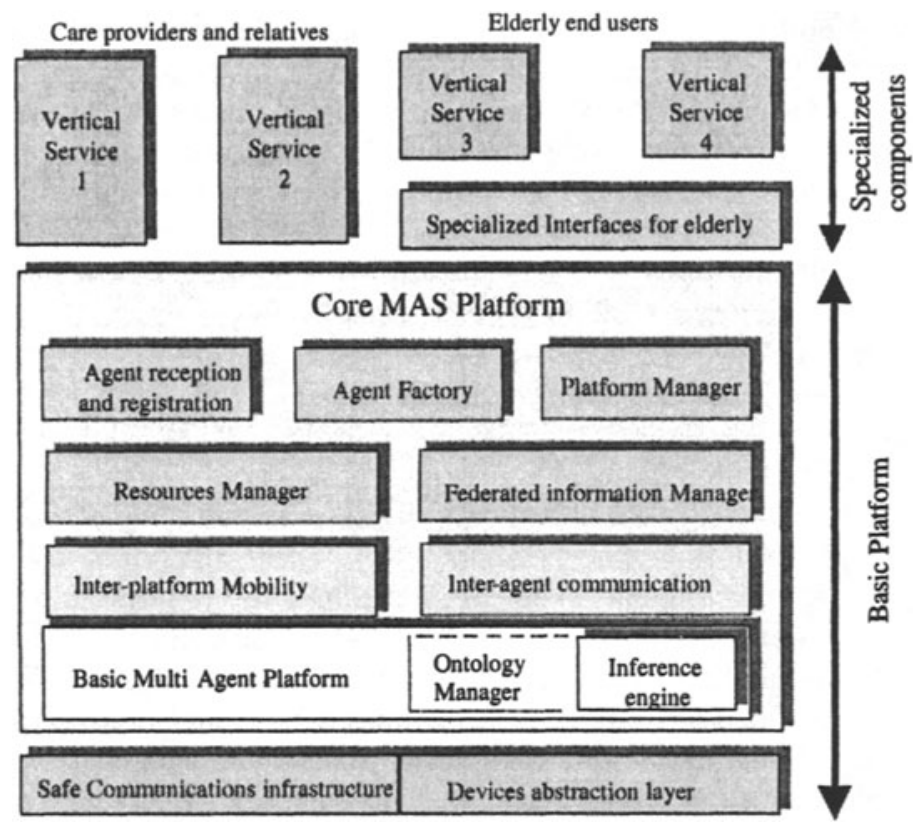

Figure 3 - Main components for an elderly virtual care community (VCC) platform

- Specialized interfaces for elderly - In the case of home sites, specialized interfaces are required for elderly that are not familiar with the use of 
computers. Intelligent interface agents resorting to multimedia services will be of invaluable importance to the system, since elderly people may present some physical and/or cultural limitations that inhibit their interaction with automatic devices such as computers, and home appliances. The ultimate goal is therefore to build the infrastructure "invisible" to the elderly. Specialized interfaces with mobile computing devices (e.g. PDAs, mobile phones) are also necessary.

Services layer - Specialized vertical services can be implemented on top of this horizontal infrastructure. Some examples include: (i) Health monitoring service and social alarm service; (ii) Medication program reminder; (iii) Home appliances operation assistant, (iv) Health-related data gathering for elderly (from distributed sources), (v) National statistics support, (v) Intelligent leisure assistant. The services can be divided in two major groups: Services for care providers and elderly relatives (that are assumed to be able to interact with normal computer interfaces), and services for elderly (which might require specialized user interfaces). These services can be implemented by combining the stationary and intelligent mobile agents with advanced federated information management mechanisms. In order to support the creation and operation of virtual communities, specific VC management services are necessary e.g. VC creation, membership rules and joining procedure, communication tools (chat, tele-conference, notice boards), but also advanced collaboration tools e.g. distributed events management, distributed (social) project management, support for elderly work (networks of retired e-lancers).

The "Basic platform" will be installed at each site (Care center, Health center, Home, etc.). For each site, a specific set of vertical services will be available (Fig. 4).

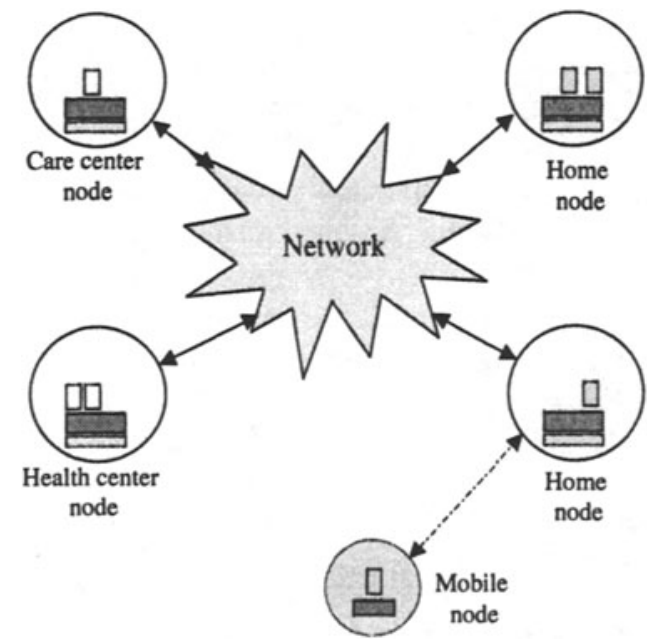

Figure 4 - TeleCARE distributed environment 
Vertical services, in the context of a multi-agent system, will be implemented by mobile and/or stationary agents. Services implemented by mobile agents may be running on mobile devices (PDAs, Mobile phones, etc), but always "linked" to a specific site.

Within the TeleCARE project, a new infrastructure supporting seamless interactions among stationary and mobile intelligent agents, with planning, error diagnosis and recovery capabilities able to recognize and self-adapt to a diversity of environments is being designed and developed. This infrastructure interoperates with the federated information management and service management environments.

The elderly care domain constitutes a highly distributed, diverse, heterogeneous and autonomous environment, that requires: advanced functionalities in terms of cooperative problem solving (for user-assistance, remote operation, status monitoring, and error recovery). Furthermore, it depends on the advanced facilities that are expected to be offered in near future by the emerging ubiquitous computing, intelligent home appliances, and dedicated appliances to assist elderly people.

Information privacy support. Considering the sensitivity of the data exchanged within this type of VC, careful preservation of the information privacy and authorized access to data is of great importance. Therefore, in the infrastructure, in addition to the user authentication and communication security, proper definition of information access rights and authorization rules are mandatory. To properly preserve the definition of adequate levels of information privacy, the VCC platform also supports a federation layer that extends the functionalities of standard database management systems. The usage of both emerging standards and Web tools (e.g. ODMG, XML, etc.) supporting the required interoperability criteria for TeleCARE is considered. On the other hand, innovative mechanisms and facilities will be developed to support the on line definition of both the syntax and semantics of "common meta-data" necessary to support the collaboration and information exchange / integration among heterogeneous and autonomous sites, with diverse goals and configurations. Furthermore, proper settings of information visibility and access rights of different sites involved in the virtual community will be supported through the federated information management layer [1], and in particular through the definition of export schemas.

Fig. 5 illustrates a proposed federated agile information management (FAIM) approach, in which three kinds of agents are considered:

- Data Interface Mapping Agent (DIMA), providing static adaptation layers for legacy information resources and/or sensor devices,

- Agent Information Management System (AIMS), Static agent at each site in charge of the federated information management,

- Mobile Information Retrieval Agent (MIRA), Federated mobile agent that performs query processing.

MIRA allows execution of federated queries among TeleCARE sites as follows: (1) User submits a federated query - Node A, (2) AIMS agent from Node A creates MIRA agents 1 and 2 with the proper sub-queries and launches them to their destinations, (3) MIRA agents retrieve information from the AIMS agents, that in turn collect information from the DIMAs, at the external sites and return to their origin site with the data, (4) The results are merged and presented to the user. 


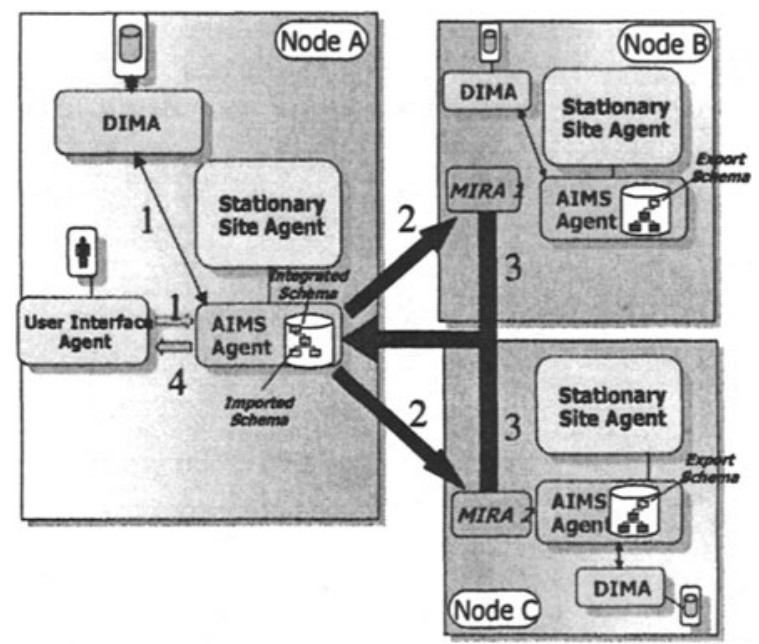

Figure 5 - Distributed information management approach

The privacy rights of the elderly will be completely preserved by the federated information management approach in the sense that the proprietary and confidential information about elderly and the centers involved can only be accessed by authorized users and centers. Furthermore, special focus is given to the security of interactions. As such, safe communications and user authentication are important complementary task in the project.

\section{FEASIBILITY PROSPECTS}

\subsection{Experiments with intelligent mobile agents for remote supervision}

A preliminary prototype focused on the adaptability of mobile agents to different environments (e.g. different home sites) was developed [5], [6]. While going through its itinerary a mobile agent has to face the heterogeneity of the environments it finds. Therefore, it is crucial that the agents have some mechanisms to cope with this heterogeneity. Also, mobile agents have to face uncertain environments that require high levels of autonomy. The concept of adaptive mobile agents that carry high level hierarchical abstract plans enabling agents to adapt themselves to the characteristics of the local environments before execution is started. These high level abstract plans are annotated with monitoring and error recovery strategies that guide the agent when errors occur. The general architecture of the mobile agents is illustrated in Fig. 6.

In this multi-agent architecture there are several places where mobile agents can reside in each moment of their lives. Each place may include several stationary agents, which implement some specific functionality within that place. There is an important distinction between the objectives of stationary agents and mobile agents. In fact, stationary agents will be used for those tasks that may only slightly change over the life of the site, while mobile agents will be used for those tasks that are not 
foreseen in advance, episodic tasks, and those tasks that have high rates of change possibilities over time. Stationary agents are involved only in cooperative work with other agents running in the same place or mobile agents that were launched from that place, while inter-place cooperation is done through mobile agents.

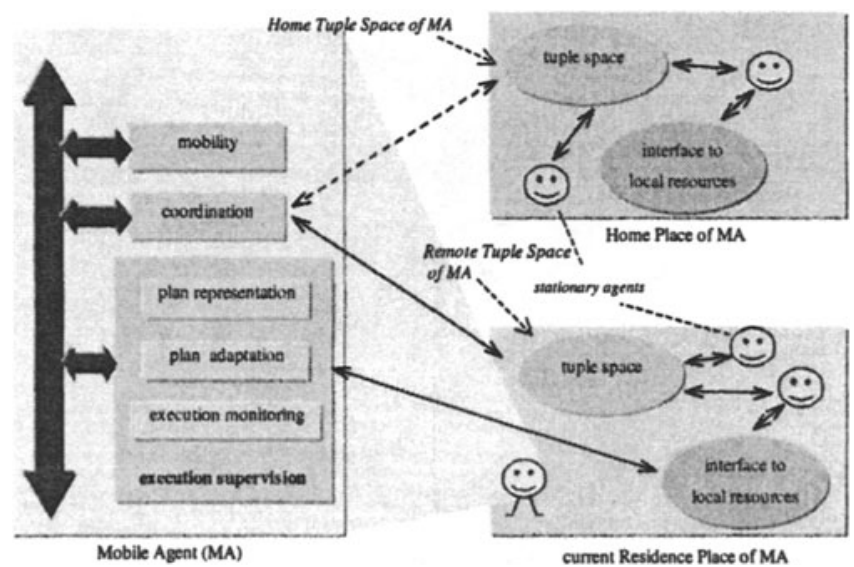

Figure 6 - General architecture of a mobile agent

The feasibility test was developed in Java, including the JINNI Prolog interpreter and was successful tested in a tele-robotics manipulation and error recovery scenario. A distributed Linda-like coordination mechanism was also implemented. Details of this experiment can be found in [5], [6] and [11].

\subsection{Inputs from VE infrastructures}

The proposed TeleCARE virtual community infrastructure benefits from the experiences and results gained within the VE area and in particular from:

- Establishment of configurable open infrastructures for VEs, like the PRODNET platform [2].

- Establishment of cooperation agreements among the various organizations and actors involved in the elderly care. Specific aspects to be borrowed from the VE area: definition of access rights to information / visibility rules, coordination policies, and safe communications.

- Support for the formation of virtual communities of elderly, complemented by low cost teleconferencing and $\mathrm{CSCW}$ tools integrated with the multi-agent infrastructure.

In the area of virtual organizations a large amount of research efforts have been devoted to the establishment of infrastructures supporting the necessary enterprises cooperation. Growing R\&D efforts are also spent on supporting the cooperation of temporary consortia of autonomous enterprises and organizations that decide to join resources and skills, in order to achieve a common goal or better respond to a business opportunity. In particular, in the case of industrial enterprises and manufacturing in specific, the field of virtual enterprises is being addressed by a 
large number of international projects [2], [3]. The results of these projects are being employed towards the design and developments in TeleCARE.

\subsection{Technology trends}

Major home appliance manufacturers are already announcing "Internet-enabled" systems. Networking technologies are starting to invade homes, not only to carry the conventional phone conversations signals and TV signals, but also the signals from surveillance cameras, commands for controlling lights and appliances, and multimedia information through the Internet. Various emerging technologies are competing in the sector of home networking, such as the wireless communications (e.g. Bluetooth), use of power lines for also transporting the information, or the structured wiring for the new constructions / new residential areas [7].

Advances on mobile computing based on the new UMTS system and its integration with other technologies such as GPS, biometric identification systems, smart cards, etc. are also important contributors that will be considered for the development of the proposed care supporting infrastructure and services.

In terms of assistive technology there are also many recent developments of portable and stationary monitoring devices.

The spread of these facilities world-wide however is a clear visible tendency of the market, and therefore facilitating the implantation of TeleCARE, when its results reach the market. There is however some uncertainty today about which one of the competing market technologies will win at the end, and when exactly the required infrastructures become generically available.

Therefore, to a great extent, the TeleCARE results will be independent of the exact winning technologies, since the system is aimed at providing a highly flexible framework. Openness is one of the main design goals for this project.

\subsection{Main difficulties}

One of the major challenges for the development of advanced care services is their acceptance by the various actors, i.e. the elderly and their relatives, the care provision assistants, health care professionals, etc.

Inclusion of advanced technology in our lives is a difficult subject as there is always the potential risk of intrusion in our privacy. Therefore, in addition to the technological developments, TeleCARE project includes tasks to study and assess its social / organizational impacts, and economic issues, training needs, ethical aspects, etc.

Furthermore, there is also the need to identify and characterize the behaviour and most adequate operating rules for this kind of virtual communities. Advanced virtual services, and even some re-designing of the basic platform, will depend on this characterization.

\section{CONCLUSIONS}

The growing numbers of elderly population impose an urgent need to develop new approaches to care provision. The convergence of a number of technologies such as 
multi-agent systems, federated information management, safe communications, hypermedia interfaces, rich sensorial environments, increased intelligence of home appliances, and collaborative virtual environments, represents an important enabling factor for the design and development of virtual elderly support community environments. In particular, a platform based on adaptive mobile agents combined with federated information management mechanisms provides a flexible infrastructure on top of which specialized care services can be built. Although these technological factors open opportunities for new approaches to elderly care, as demonstrated by preliminary research results, this process has to be complemented by a careful analysis of the socio-economic impacts and ethical issues.

Acknowledgements. This work was funded in part by the IST program of the European Commission. The authors thank the contribution of the TeleCARE consortium.

\section{REFERENCES}

1. Afsarmanesh, H.; Garita, C; Hertzberger, L.O. - Virtual Enterprises and Federated Information Sharing. Proceedings of the 9th IEEE Int. Conf. on Database and Expert Systems Applications", DEXA'98, Vienna, Austria, Aug 1998.

2. Camarinha-Matos, L.M.; Afsarmanesh, H. - Infrastructures for Virtual Enterprises, Kluwer Academic Publishers, ISBN 0-7923-8639-6, Oct. 1999.

3. Camarinha-Matos, L.M.; Afsarmanesh, H.; Rabelo, R. - E-Business and Virtual Enterprises, Kluwer Academic Publishers, ISBN 0-7923-7205-0, Oct 2000.

4. Camarinha-Matos, L.M.; Afsarmanesh, H. - Virtual communities and elderly support, MIV'01 in "Advances in Automation, Multimedia and Video Systems, and Modern Compuuter Science", (V.V. Kluev, C.E. D'Attellis, N. E. Mastorakis ed.s), WSES, ISBN 960-8052-44-0, pp. 279-284, Sept 2001.

5. Camarinha-Matos, L.M.; Vieira, W. - Intelligent mobile agents in elderly care, Journal of Robotics and Autonomous Systems (Elsevier), Vol. 27, N. 1-2, April 1999, ISSN 0921-8890, pp. 59-75.

6. Camarinha-Matos, L.M.; Vieira, W.; Castolo, O. - Mobile agents approach to virtual laboratories, Proceedings of IFAC Symposium on Artificial Intelligence in Real-Time Control, Budapest, 2-4 Oct 2000.

7. Dutta-Roy, A. - Networks for homes, IEEE Spectrum, Vol. 36, N. 12, Dec 1999.

8. Saranummi, N.; Kivisaari, S.; Sarkikoski, T.; Graafmans, J. - Ageing \& Technology State of the art, Report for the European Commission, Institute for Prospective Studies, Seville, Spain, Nov. 1996.

9. Telecare Consortium - A Multi-Agent Tele-Supervision System for Elderly Care, Description of work, Jan 2001

10. Vlaskamp, F. - Social alarms go mobile: emergency assistance for mobile users, 1999, www.fernuni-hagen.de/FTB/aaate99/paper/99 67.htm.

11. Vieira, W.; Camarinha-Matos, L.M.; Castolo, O. - Fitting autonomy and mobile agents, Proceedings of ETFA'01 - 8th IEEE International Conference on Emerging Technologies and Factory Automation Vol. 2, IEEE Catalog Number 01TH8597, pp. 471-480, Antibes - Juan les Pins, France, Oct 15-18, 2001. 